\title{
Error Exponents in Scalable Source Coding
}

\author{
Ertem Tuncel and Kenneth Rose \\ $\{$ ertem,rose\}ece.ucsb.edu \\ Dept. of ECE, University of California, Santa Barbara, CA 93106
}

\begin{abstract}
We extend the error exponent results to 2layer scalable source coding. We consider separate error events at each layer so as to allow a trade-off analysis for the error exponents when the rate and distortion values are fixed. Given a discrete memoryless source, we derive the single-letter characterization of the region of all achievable 6-tuples $\left(R_{1}, R_{2}, D_{1}, D_{2}, E_{1}, E_{2}\right)$, i.e., the rate, distortion, and error exponent levels at each layer. We also analyze the special case of successive refinability, where the triplets $\left(R_{1}, D_{1}, E_{1}\right)$ and $\left(R_{2}, D_{2}, E_{2}\right)$ individually achieve the nonscalable bounds. It is surprising that for any $D_{1}, D_{2}$, and $R_{1}$, there exists an $\hat{R}_{2}$ such that successive refinability is ensured for all $R_{2} \geq \hat{R}_{2}$.
\end{abstract}

Keywords - Scalable source coding, error exponents, successive refinement.

\section{INTRODUCTION}

The rate-distortion function $R(D)$ indicates the minimum rate required to (asymptotically) achieve an average distortion $D$. A more demanding rate-distortion problem arises from statistical consideration of the error event, i.e., the event that a source vector is compressed at distortion exceeding $D$. While the rate $R(D)$ is sufficient to ensure that the error probability vanishes as the block length $n$ tends to infinity, a major concern is with its asymptotic rate of decay. The asymptotic decay is typically quantified by the error exponent $E=-\frac{1}{n} \log \operatorname{Pr}$ [error]. Thus, the rate-distortion problem may be generalized to ask one of the two questions: (i) What is the minimum rate required to achieve an error exponent at or above a given level? (ii) What is the maximum error exponent achievable at or below a given coding rate? The standard rate-distortion problem corresponds to the special case of (i) with required error exponent $E \longrightarrow 0$.

The best error exponent for nonscalable source coding was first characterized by Marton [7]. Given a discrete memoryless source (DMS) with distribution $P$, and given distortion and rate levels $D$ and $R$, respectively, the best error exponent is

$$
E_{P}(D, R)=\inf _{P^{\prime}: R<R_{P^{\prime}}(D)} \mathcal{D}\left(P^{\prime} \| P\right),
$$

where $\mathcal{D}\left(P^{\prime} \| P\right)$ is the information divergence and $R_{P}(D)$ is the rate-distortion function for source $P$, which is given by

$$
R_{P}(D)=\min _{Q(y \mid x): E_{P, Q}\{d(X, Y)\} \leq D} I_{P, Q}(X ; Y) .
$$

* This work was supported in part by the NSF under grant no. MIP-9707764, EIA 9986057, the UC MICRO program, Conexant Systems, Inc., Dolby Laboratories, Inc., Lucent Technologies, Inc., Media Stream, Inc., and Qualcomm, Inc.
This result is valid under the condition $R \geq R_{P}(D)$. Marton also discussed the existence of discontinuities in $E_{P}(D, R)$ as a function of $R$, possibly for a countable set of rates. Sufficient conditions for continuity of $E_{P}(D, R)$ for all $R$ were derived in [7] and [1].

In this paper, we extend these results to 2-layer scalable coding, and derive a characterization for $E_{P}\left(D_{1}, D_{2}, R_{1}, R_{2}, E_{1}\right)$, the best error exponent achievable in the second layer given the distortion and rate constraints for both layers, and the error exponent constraint for the first layer. Kanlis and Narayan [6] previously considered an extension of the nonscalable result, however they defined as error the event that either the first layer distortion exceeds $D_{1}$, or the second layer distortion exceeds $D_{2}$, precluding a possible trade-off analysis between the exponents of the two error probabilities. Haroutunian et al. [5] analyzed the special "successive refinability" case, i.e., the conditions under which

$$
E_{P}\left(D_{1}, D_{2}, R_{1}, R_{2}, E_{P}\left(D_{1}, R_{1}\right)\right)=E_{P}\left(D_{2}, R_{2}\right)
$$

is satisfied. We further use our characterization of the function $E_{P}\left(D_{1}, D_{2}, R_{1}, R_{2}, E_{1}\right)$ to analyze the special case of successive refinability, and prove a necessary and sufficient condition which is fundamentally different from the condition in [5]. In particular, it implies that for every $D_{1}, D_{2}$, and $R_{1}$, there exists a $\hat{R}_{2}$ such that successive refinability is ensured for all $R_{2} \geq \hat{R}_{2}$.

We begin with some preliminaries in the following section. In Section 3, we employ the type covering lemmas [3], [6], to construct a coding strategy and in Section 4 we prove, by extending the approach of [7], that no better coding strategy exists. Finally, in Section 5, we analyze the special case of successive refinability.

\section{Preliminaries And Basic Definitions}

We denote the source and the reproduction alphabets by $\mathcal{X}$ and $\hat{\mathcal{X}}$, respectively. We assume a single-letter distortion measure $d: \mathcal{X} \times \hat{\mathcal{X}} \rightarrow[0, \infty)$, i.e., the distortion measure extends to $n$ dimensions as $d\left(x^{n}, \hat{x}^{n}\right)=$ $\frac{1}{n} \sum_{i=1}^{n} d\left(x_{i}, \hat{x}_{i}\right)$. In the scalable coding scenario, for generality, we allow for two layer-specific single-letter distortion measures $d_{1}$ and $d_{2}$. We denote by $|f|$ the cardinality of the range of a function $f$.

Definition 1: $(R, D, E)$ is called an achievable ratedistortion-exponent triplet if for any given $\delta>0$, there exist an encoding function

$$
f: \mathcal{X}^{n} \longrightarrow\left\{1,2, \ldots, 2^{n R}\right\},
$$


and a decoding function

$$
g:\left\{1,2, \ldots, 2^{n R}\right\} \longrightarrow \hat{\mathcal{X}}^{n},
$$

such that

$$
-\frac{1}{n} \log \operatorname{Pr}\left[d\left(X^{n}, g\left(f\left(X^{n}\right)\right)\right)>D\right]>E-\delta
$$

for all $n \geq n_{0}(\delta)$.

From (1), it is clear that for a source $P$, and for $E>0$, $(R, D, E)$ is achievable if and only if

$$
R \geq R_{P}(D) \text { and } \inf _{P^{\prime}: R<R_{P^{\prime}}(D)} \mathcal{D}\left(P^{\prime} \| P\right)>E,
$$

or in other words, if and only if

$$
\forall P^{\prime}: \mathcal{D}\left(P^{\prime} \| P\right) \leq E \Longrightarrow R \geq R_{P^{\prime}}(D) .
$$

Definition 2: The rate-distortion-exponent function, $R_{P}(D, E)$, is defined as the minimum $R$ such that $(R, D, E)$ is achievable for source $P$.

The condition (4) implies that

$$
R_{P}(D, E)=\sup _{P^{\prime}: \mathcal{D}\left(P^{\prime} \| P\right) \leq E} R_{P^{\prime}}(D) .
$$

In the special case of $E \longrightarrow 0,(R, D, E)$ is achievable if and only if $R \geq R_{P}(D)$ is satisfied. Another extreme case is when $E \longrightarrow \infty$, for which (5) yields $R^{0}(D)$, i.e., the "zero-error" rate-distortion function [3, Theorem 2.4.2].

Definition 3: $\left(R_{1}, R_{2}, D_{1}, D_{2}, E_{1}, E_{2}\right)$ with $D_{2} \leq D_{1}$ and $R_{2} \geq R_{1}$ is called an achievable 2-stage ratedistortion-exponent 6 -tuple if for any given $\delta_{1}>0$ and $\delta_{2}>0$, there exist stage-encoding functions

$$
\begin{gathered}
f_{1}: \mathcal{X}^{n} \longrightarrow\left\{1,2, \ldots, 2^{n R_{1}}\right\} \\
f_{2}: \mathcal{X}^{n} \longrightarrow\left\{1,2, \ldots, 2^{n\left[R_{2}-R_{1}\right]}\right\}
\end{gathered}
$$

and stage-decoding functions

$$
\begin{gathered}
g_{1}:\left\{1,2, \ldots, 2^{n R_{1}}\right\} \longrightarrow \hat{\mathcal{X}}^{n} \\
g_{2}:\left\{1,2, \ldots, 2^{n R_{1}}\right\} \times\left\{1,2, \ldots, 2^{n\left[R_{2}-R_{1}\right]}\right\} \longrightarrow \hat{\mathcal{X}}^{n},
\end{gathered}
$$

such that

$$
-\frac{1}{n} \log \operatorname{Pr}\left[d_{1}\left(X^{n}, g_{1}\left(f_{1}\left(X^{n}\right)\right)\right)>D_{1}\right]>E_{1}-\delta_{1}
$$

and

$$
-\frac{1}{n} \log \operatorname{Pr}\left[d_{2}\left(X^{n}, g_{2}\left(f_{1}\left(X^{n}\right), f_{2}\left(X^{n}\right)\right)\right)>D_{2}\right]>E_{2}-\delta_{2}
$$

for all $n \geq n_{0}\left(\delta_{1}, \delta_{2}\right)$.

The special case $E_{1}, E_{2} \longrightarrow 0$ corresponds to Rimoldi's successive refinement characterization [8], as the 6-tuple $\left(R_{1}, R_{2}, D_{1}, D_{2}, E_{1}, E_{2}\right)$ becomes achievable if and only if $R_{1} \geq R_{P}\left(D_{1}\right)$ and $R_{2} \geq R_{P}\left(D_{1}, D_{2}, R_{1}\right)$, where $R_{P}\left(D_{1}, D_{2}, R_{1}\right)$ is given by

$$
R_{P}\left(D_{1}, D_{2}, R_{1}\right)=\min _{\substack{(y, z \mid x): I_{P, Q}(X ; Y) \\ E_{P, Q}\left\{d_{1}(X, Y)\right\} \\ E_{P, Q}\left\{d_{2}(X, Z)\right\}}} I_{P, Q} \leq D_{1}(X ; Y, Z)
$$

if $R_{1} \leq R_{P}\left(D_{2}\right)$, and

$$
R_{P}\left(D_{1}, D_{2}, R_{1}\right)=R_{1}
$$

otherwise $^{1}$.

Definition 4: Given source $P$, distortion levels $D_{1} \geq$ $D_{2}$, and rate $R_{1} \geq R_{P}\left(D_{1}, E_{1}\right)$, the scalable rate-distortion-exponent function $R_{P}\left(D_{1}, D_{2}, R_{1}, E_{1}, E_{2}\right)$ is defined as the minimum $R_{2}$ such that $\left(R_{1}, R_{2}, D_{1}, D_{2}, E_{1}, E_{2}\right)$ is achievable.

Definition 5: Similarly, the scalable error exponent function $E_{P}\left(D_{1}, D_{2}, R_{1}, R_{2}, E_{1}\right)$ for source $P$, defined under the conditions $D_{1} \geq D_{2}, R_{1} \geq R_{P}\left(D_{1}, E_{1}\right)$, and $R_{2} \geq R_{P}\left(D_{1}, D_{2}, R_{1}\right)$, is the minimum $E_{2}$ such that $\left(R_{1}, R_{2}, D_{1}, D_{2}, E_{1}, E_{2}\right)$ is achievable.

\section{Sufficient Conditions for Achievability}

We derive sufficient conditions for achievability by constructing an actual scalable coding strategy. To this end, we employ the type covering lemma [3], and its scalable extension proved by Kanlis and Narayan [6]. The strategy exploits a fundamental property of types: the number of distinct types for sequences of length $n$ grows at most polynomially with $n$. Hence, we may tailor encoding functions to each type separately, without compromising the overall coding rate asymptotically [3].

We denote by $T_{P}^{n}$ the set of all source vectors $x^{n}$ having type $P$. We separately analyze the two cases $E_{1}<E_{2}$ and $E_{1} \geq E_{2}$. Note that in order for $\left(R_{1}, R_{2}, D_{1}, D_{2}, E_{1}, E_{2}\right)$ to be achievable, it is necessary to satisfy condition (4) for the first layer:

$$
\forall P^{\prime}: \mathcal{D}\left(P^{\prime} \| P\right) \leq E_{1} \Longrightarrow R_{1} \geq R_{P^{\prime}}\left(D_{1}\right) .
$$

Case I: $E_{1}<E_{2}$.

We adopt the following strategy: For type $P^{\prime}$ :

- If $\mathcal{D}\left(P^{\prime}|| P\right) \leq E_{1}$, then we can generate $2^{n R_{1}}$ balls of radius $D_{1}$ in the first layer, and for each $D_{1}$-ball, generate $2^{n\left[R_{P^{\prime}}\left(D_{1}, D_{2}, R_{1}\right)-R_{1}\right]}$ balls of radius $D_{2}$ in the second layer, such that for every source vector $x^{n} \in T_{P^{\prime}}^{n}$, there exists a pair of $D_{1^{-}}$and $D_{2}$-balls covering $x^{n}$. Since $R_{1} \geq R_{P^{\prime}}\left(D_{1}\right)$ from (8), this 2 layer covering is indeed possible for large $n$, as proved in $[6]$.

- If $E_{1}<\mathcal{D}\left(P^{\prime} \| P\right) \leq E_{2}$, then we cover $T_{P^{\prime}}^{n}$ with $2^{n R_{P^{\prime}}\left(D_{2}\right)}$ balls, only for the purpose of using the ball centers as second layer codevectors. In the first stage, we send the first $R_{1}$ bits, and do not reproduce anything at the decoder, and in the second stage send the rest of the bits (if any), and reproduce the ball centers at the decoder.

- If $E_{2}<\mathcal{D}\left(P^{\prime} \| P\right)$, then we do not perform any covering.

${ }^{1}$ Observe that if $R_{1}>R_{P}\left(D_{2}\right)$, the minimum in (6) is $R_{P}\left(D_{2}\right)$, which makes $R_{1}$ greater than the achieved minimum. On the other hand, if $R_{1} \leq R_{P}\left(D_{2}\right)$, then the minimum in (6) is always greater than or equal to $R_{P}\left(D_{2}\right)$. 


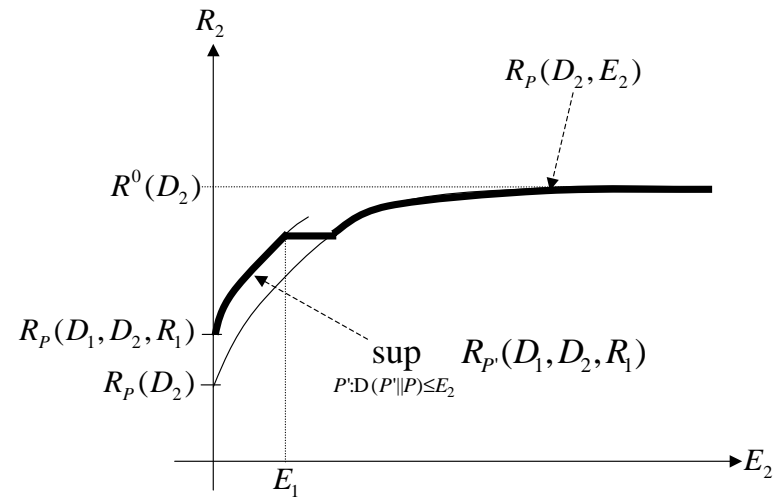

Fig. 1. A typical curve of achievable $R_{2}$ versus $E_{2}$, given fixed $D_{1}$, $D_{2}, R_{1}$, and $E_{1}$, where $R_{1} \geq R_{P}\left(D_{1}, E_{1}\right)$.

It is clear that the first and second-layer error exponents are at least $E_{1}$, and $E_{2}$, respectively. The achieved rate at the second layer is

$$
\begin{aligned}
R_{2}=\max & \left\{\sup _{P^{\prime}: E_{1}<\mathcal{D}\left(P^{\prime} \| P\right) \leq E_{2}} R_{P^{\prime}}\left(D_{2}\right),\right. \\
& \left.\sup _{P^{\prime}: \mathcal{D}\left(P^{\prime} \| P\right) \leq E_{1}} R_{P^{\prime}}\left(D_{1}, D_{2}, R_{1}\right)\right\} .
\end{aligned}
$$

or in a more compact form

$R_{2}=\max \left\{R_{P}\left(D_{2}, E_{2}\right), \sup _{P^{\prime}: \mathcal{D}\left(P^{\prime} \| P\right) \leq E_{1}} R_{P^{\prime}}\left(D_{1}, D_{2}, R_{1}\right)\right\}$

since $R_{P^{\prime}}\left(D_{1}, D_{2}, R_{1}\right) \geq R_{P^{\prime}}\left(D_{2}\right)$ for all $P^{\prime}$.

Case II: $E_{1} \geq E_{2}$.

We adopt the following strategy: For type $P^{\prime}$ :

- If $\mathcal{D}\left(P^{\prime} \| P\right) \leq E_{2}$, then similarly to the first case, we perform a two-layer covering of type $P^{\prime}$ using $2^{n R_{1}}$ balls of radius $D_{1}$ in the first layer, and $2^{n R_{P^{\prime}}\left(D_{1}, D_{2}, R_{1}\right)}$ balls of radius $D_{2}$ in the second layer. Since $\mathcal{D}\left(P^{\prime} \| P\right) \leq E_{2} \leq E_{1}$, it follows from (8) that $R_{1} \geq R_{P^{\prime}}\left(D_{1}\right)$, and hence this 2-layer covering is again possible.

- If $E_{2}<\mathcal{D}\left(P^{\prime} \| P\right) \leq E_{1}$, then we cover $T_{P^{\prime}}^{n}$ with $2^{n R_{P^{\prime}}\left(D_{1}\right)}$ balls, only for the purpose of using the ball centers as first layer codevectors.

- If $E_{1}<\mathcal{D}\left(P^{\prime} \| P\right)$, then we do not perform any covering.

It is easily verified that the first and second-layer error exponents are at least $E_{1}$, and $E_{2}$, respectively. The achieved rate at the second layer is given by

$$
R_{2}=\sup _{P^{\prime}: \mathcal{D}\left(P^{\prime} \| P\right) \leq E_{2}} R_{P^{\prime}}\left(D_{1}, D_{2}, R_{1}\right) .
$$

Combining (9) and (11), we observe that if $R_{2} \geq$ $R^{*}\left(D_{1}, D_{2}, R_{1}, E_{1}, E_{2}\right)$, where $R^{*}\left(D_{1}, D_{2}, R_{1}, E_{1}, E_{2}\right)$ is given in (12) at the bottom, then $\left(R_{1}, R_{2}, D_{1}, D_{2}, E_{1}, E_{2}\right)$ is achievable. However, for the purpose of the proof that $R^{*}\left(D_{1}, D_{2}, R_{1}, E_{1}, E_{2}\right)$ actually specifies the entire achievable region, it will be more convenient to work with infimum of $\mathcal{D}\left(P^{\prime} \| P\right)$ over certain sets. The corresponding sufficient condition for achievability is given by

$$
E_{2} \leq E^{*}\left(D_{1}, D_{2}, R_{1}, R_{2}, E_{1}\right),
$$

where $E^{*}\left(D_{1}, D_{2}, R_{1}, R_{2}, E_{1}\right)$ is defined as in (13) with the standard convention that infimum over an empty set yields infinity. A fairly general curve of the achieved $R_{2}$ with this coding strategy is shown in Figure 1.

\section{Necessary Conditions for Achievability}

For any coding strategy given by encoding and decoding functions $f_{1}, f_{2}, g_{1}$, and $g_{2}$, as described in Definition 3 , we introduce the notation

$$
\mathcal{U}_{1}\left(f_{1}, g_{1}\right)=\left\{x^{n}: d_{1}\left(x^{n}, g_{1}\left(f_{1}\left(x^{n}\right)\right)\right)>D_{1}\right\},
$$

for the set of points in $\mathcal{X}^{n}$ that are not reproduced within distortion $D_{1}$ at the first stage. Similarly, the set of points that are not reproduced within distortion $D_{2}$ at the second stage is denoted by

$$
\mathcal{U}_{2}\left(f_{1}, f_{2}, g_{2}\right)=\left\{x^{n}: d_{2}\left(x^{n}, g_{2}\left(f_{1}\left(x^{n}\right), f_{2}\left(x^{n}\right)\right)\right)>D_{2}\right\} .
$$

Theorem 1: Given a discrete source with probability distribution $P$, let $R_{1} \geq R_{P}\left(D_{1}, E_{1}\right)$. A coding strategy given by $f_{1}, f_{2}, g_{1}$, and $g_{2}$ satisfies

$$
\begin{aligned}
\frac{1}{n} \log \left|f_{1}\right| & <R_{1} \\
\frac{1}{n} \log \left|f_{1} \times f_{2}\right| & <R_{2} \\
-\frac{1}{n} \log P^{n}\left(\mathcal{U}_{1}\left(f_{1}, g_{1}\right)\right) & >E_{1}-\delta_{1}
\end{aligned}
$$

for any given $\delta_{1}>0$, and for all $n \geq n_{0}\left(\delta_{1}\right)$ only if

$$
\begin{gathered}
\limsup _{n \rightarrow \infty}\left\{-\frac{1}{n} \log P^{n}\left(\mathcal{U}_{2}\left(f_{1}, f_{2}, g_{2}\right)\right)\right\} \\
\leq E^{*}\left(D_{1}, D_{2}, R_{1}, R_{2}, E_{1}-\delta_{1}\right) .
\end{gathered}
$$

$$
\begin{aligned}
R^{*}\left(D_{1}, D_{2}, R_{1}, E_{1}, E_{2}\right) & \triangleq \max \left\{R_{P}\left(D_{2}, E_{2}\right), \sup _{P^{\prime}: \mathcal{D}\left(P^{\prime} \| P\right) \leq \min \left(E_{1}, E_{2}\right)} R_{P^{\prime}}\left(D_{1}, D_{2}, R_{1}\right)\right\} \\
E^{*}\left(D_{1}, D_{2}, R_{1}, R_{2}, E_{1}\right) & \triangleq \min \left\{E_{P}\left(D_{2}, R_{2}\right), \inf _{\substack{P^{\prime}: \mathcal{D}\left(P^{\prime} \| P\right) \leq E_{1} \text { and } \\
R_{2}<R_{P^{\prime}}\left(D_{1}, D_{2}, R_{1}\right)}} \mathcal{D}\left(P^{\prime} \| P\right)\right\}
\end{aligned}
$$


Remark: It follows from the theorem that the achievable region constructed in the previous section is the largest possible achievable region. In other words,

$$
\begin{aligned}
& E_{P}\left(D_{1}, D_{2}, R_{1}, R_{2}, E_{1}\right)=E^{*}\left(D_{1}, D_{2}, R_{1}, R_{2}, E_{1}\right) \\
& R_{P}\left(D_{1}, D_{2}, R_{1}, E_{1}, E_{2}\right)=R^{*}\left(D_{1}, D_{2}, R_{1}, E_{1}, E_{2}\right) .
\end{aligned}
$$

Sketch of Proof: First, we define three sets

$$
\begin{aligned}
& \mathcal{Q}_{1}=\left\{Q: \mathcal{D}(Q \| P)<E_{1}-\delta_{1}\right\} \\
& \mathcal{Q}_{2}=\left\{Q: R_{2}<R_{Q}\left(D_{1}, D_{2}, R_{1}\right)\right\} \\
& \mathcal{Q}_{3}=\left\{Q: R_{2}<R_{Q}\left(D_{2}\right)\right\} .
\end{aligned}
$$

We observe that for any $Q \in \mathcal{Q}_{1}, Q^{n}\left(\mathcal{U}_{1}\left(f_{1}, g_{1}\right)\right) \longrightarrow 0$ as $n \longrightarrow \infty$. Using this observation, we prove that for all $Q \in \mathcal{Q}_{3} \cup\left(\mathcal{Q}_{1} \cap \mathcal{Q}_{2}\right)$, there exists a constant $\alpha\left(Q, D_{1}, D_{2}, R_{1}, R_{2}, E_{1}\right)>0$ such that

$$
Q^{n}\left(\mathcal{U}_{2}\left(f_{1}, f_{2}, g_{2}\right)\right) \geq \alpha\left(Q, D_{1}, D_{2}, R_{1}, R_{2}, E_{1}\right) .
$$

Next, we define

$$
G^{n} \triangleq\left\{x^{n}:\left|\frac{1}{n} \log \frac{Q^{n}\left(x^{n}\right)}{P^{n}\left(x^{n}\right)}-\mathcal{D}(Q \| P)\right|<\eta\right\} .
$$

The weak law of large numbers ensures that

$$
Q^{n}\left(G^{n}\right)>1-\frac{1}{2} \alpha\left(Q, D_{1}, D_{2}, R_{1}, R_{2}, E_{1}\right)
$$

for all $Q \in \mathcal{Q}_{3} \cup\left(\mathcal{Q}_{1} \cap \mathcal{Q}_{2}\right)$, for sufficiently large $n$. We next consider the error probability at the second layer:

$$
\begin{aligned}
P^{n} & \left(\mathcal{U}_{2}\left(f_{1}, f_{2}, g_{2}\right)\right) \\
\geq & P^{n}\left(\mathcal{U}_{2}\left(f_{1}, f_{2}, g_{2}\right) \cap G^{n}\right) \\
= & \sum_{x^{n} \in \mathcal{U}_{2}\left(f_{1}, f_{2}, g_{2}\right) \cap G^{n}} P^{n}\left(x^{n}\right) \\
= & \sum_{x^{n} \in \mathcal{U}_{2}\left(f_{1}, f_{2}, g_{2}\right) \cap G^{n}} Q^{n}\left(x^{n}\right) \exp \left\{-\log \frac{Q^{n}\left(x^{n}\right)}{P^{n}\left(x^{n}\right)}\right\} \\
\geq & Q^{n}\left(\mathcal{U}_{2}\left(f_{1}, f_{2}, g_{2}\right) \cap G^{n}\right) \exp \{-n[\mathcal{D}(Q \| P)+\eta]\} \\
\geq & \frac{1}{2} \alpha\left(Q, D_{1}, D_{2}, R_{1}, R_{2}, E_{1}\right) \exp \{-n[\mathcal{D}(Q \| P)+\eta]\}
\end{aligned}
$$

for sufficiently large $n$, where the last inequality follows from (15) and (16). This implies that

$$
\limsup _{n \rightarrow \infty}\left\{-\frac{1}{n} \log P^{n}\left(\mathcal{U}_{2}\left(f_{1}, f_{2}, g_{2}\right)\right)\right\} \leq \mathcal{D}(Q \| P)+\eta
$$

for all $Q \in \mathcal{Q}$ and all $\eta$. The result follows after taking the infimum of both sides over the set $\mathcal{Q}$, and letting $\eta \longrightarrow 0$.

\section{Successive Refinability}

Let $\hat{R}_{2}$ be defined as

$$
\hat{R}_{2}=\sup _{P^{\prime}: \mathcal{D}\left(P^{\prime} \| P\right) \leq E_{P}\left(D_{1}, R_{1}\right)} R_{P^{\prime}}\left(D_{1}, D_{2}, R_{1}\right) .
$$

Pictorially, $\hat{R}_{2}$ corresponds to the straight line in Figure 1 when $E_{1}$ is set to $E_{P}\left(D_{1}, R_{1}\right)$. It follows from (13) that successive refinement is achievable, i.e.,

$$
E_{P}\left(D_{1}, D_{2}, R_{1}, R_{2}, E_{P}\left(D_{1}, R_{1}\right)\right)=E_{P}\left(D_{2}, R_{2}\right)
$$

at all second layer rates $R_{2} \geq \hat{R}_{2}$. If $R_{2}<\hat{R}_{2}$, then by (13), successive refinability requires

$$
\inf _{P^{\prime}: \mathcal{D}\left(P^{\prime} \| P\right) \leq E_{P}\left(D_{1}, R_{1}\right) \text { and }} \mathcal{D}\left(P^{\prime} \| P\right)=E_{P}\left(D_{2}, R_{2}\right) .
$$

The successive refinability condition for the case $R_{2}<\hat{R}_{2}$ may be restated equivalently as

$$
\begin{aligned}
E_{P}\left(D_{1}, R_{1}\right) & \geq E_{P}\left(D_{2}, R_{2}\right) \\
R_{P^{*}}\left(D_{1}, D_{2}, R_{1}\right) & =R_{P^{*}}\left(D_{2}\right)
\end{aligned}
$$

where distribution $P^{*}$ achieves the infimum in (18). Note that for the special case $R_{1}=R_{P}\left(D_{1}\right)$ and $R_{2}=R_{P}\left(D_{2}\right)$, it follows that $E_{P}\left(D_{1}, R_{1}\right)=E_{P}\left(D_{2}, R_{2}\right)=0$ and the above conditions for successive refinability reduce to

$$
R_{P}\left(D_{2}\right)=R_{P}\left(D_{1}, D_{2}, R_{P}\left(D_{1}\right)\right),
$$

for which the necessary and sufficient condition is the well-known Markovian condition given in [4].

\section{CONCLUSION}

We characterized the region of all achievable 6 -tuples $\left(R_{1}, R_{2}, D_{1}, D_{2}, E_{1}, E_{2}\right)$ for the scalable source coding scenario. Given source $P$, the characterization is in terms of the information divergence $\mathcal{D}\left(P^{\prime} \| P\right)$ and the rate distortion functions $R_{P^{\prime}}\left(D_{2}\right)$ and $R_{P^{\prime}}\left(D_{1}, D_{2}, R_{1}\right)$, for all sources $P^{\prime}$. We specialized the necessary and sufficient achievability conditions to the successive refinability case, and obtained the surprising result that it is possible to achieve the bounds $E_{1}=E_{P}\left(D_{1}, R_{1}\right)$, and $E_{2}=E_{P}\left(D_{2}, R_{2}\right)$, for all second layer rates $R_{2}$ above a specified threshold.

\section{REFERENCES}

[1] R. Ahlswede. Extremal properties of rate-distortion functions. IEEE Trans. on Information Theory 36(1):166-171, January 1990.

[2] T. M. Cover and J. A. Thomas. Elements of Information Theory. Wiley, New York, 1991.

[3] I. Csiszár. Information Theory. Coding Theorems for Discrete Memoryless Sources. Akadémiai Kiadó, Budapest, Hungary, 1981.

[4] W. H. R. Equitz and T. M. Cover. Successive refinement of information. IEEE Trans. on Information Theory 37(2):269275, March 1991.

[5] E. A. Haroutunian and A. N. Harutyunyan. Successive refinement of information with reliability criterion IEEE International Symposium on Information Theory, page 205, Sorrento, Italy, June 2000.

[6] A. Kanlis and P. Narayan. Error exponents for successive refinement by partitioning. IEEE Trans. on Information Theory, 42(1):275-282, January 1996.

[7] K. Marton. Error exponent for source coding with a fidelity criterion. IEEE Trans. on Information Theory, 20(2):197-199, March 1974.

[8] B. Rimoldi. Successive refinement of information: characterization of the achievable rates. IEEE Trans. on Information Theory, 40(1):253-259, January 1994. 